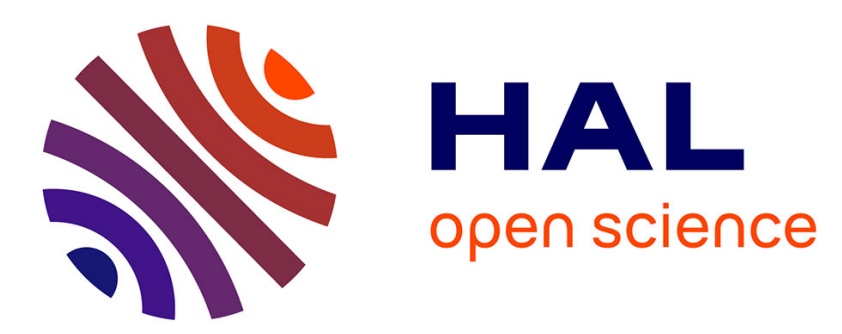

\title{
Une méthode de calcul de l'évapotranspiration réelle à partir de mesures ponctuelles de résistance stomatique et de température foliaire
}

\author{
Jean-Paul Lhomme, Nader Katerji
}

\section{- To cite this version:}

Jean-Paul Lhomme, Nader Katerji. Une méthode de calcul de l'évapotranspiration réelle à partir de mesures ponctuelles de résistance stomatique et de température foliaire. Agronomie, 1986, 5 (5), pp.397-403. hal-00884824

\section{HAL Id: hal-00884824 \\ https://hal.science/hal-00884824}

Submitted on 1 Jan 1986

HAL is a multi-disciplinary open access archive for the deposit and dissemination of scientific research documents, whether they are published or not. The documents may come from teaching and research institutions in France or abroad, or from public or private research centers.
L'archive ouverte pluridisciplinaire HAL, est destinée au dépôt et à la diffusion de documents scientifiques de niveau recherche, publiés ou non, émanant des établissements d'enseignement et de recherche français ou étrangers, des laboratoires publics ou privés. 


\title{
Une méthode de calcul de l'évapotranspiration réelle à partir de mesures ponctuelles de résis- tance stomatique et de température foliaire
}

\author{
Jean-Paul LHOMME \& Nader KATERJI (*) \\ I.N.A.P.G., Chaire de Bioclimatologie, 16, rue Claude-Bernard, F 75005 Paris \\ (*) I.N.R.A., Station de Bioclimatologie, route de Saint-Cyr, F 78000 Versailles
}

\begin{abstract}
Nous proposons un modèle analogique qui permet le calcul de l'évapotranspiration globale d'un couvert végétal à partir des mesures ponctuelles de résistance stomatique et de température foliaire. Ce modèle est basé sur une division du couvert en différentes strates, chacune caractérisee par deux températures $T_{r_{i}}$ et $T s_{i}$, et deux résistances élémentaires $r_{a_{i}}$ et re $r_{i}$ (fig. 2 et 3 ). $T r_{i}$ représente la température de rosée de l'air de la strate $i$ et $\mathrm{Ts}_{\mathrm{i}}{ }_{\mathrm{ia}}$ température moyenne des surfaces foliaires ; $\mathrm{ra}_{\mathrm{i}}$ caractérise la résistance à la diffusion de la vapeur d'eau au travers de la strate $i$ et $\mathrm{r}_{\mathrm{i}}$ la résistance à la diffusion des cavités stomatiques jusqu'au sommet de la strate (re $_{\mathrm{i}}$ englobant la résistance stomatique) (fig. 1). Le modèle conduit à une expression mathématique du flux global d'évapotranspiration fonction de la température de rosée de l'air au sommet du couvert $\operatorname{Tr}\left(\mathrm{z}_{\mathrm{h}}\right)$, des températures des surfaces foliaires $T s_{i}$ et des résistances élémentaires $r_{i}$ et re $\mathrm{i}_{\mathrm{i}}$. Le modèle est testé avec des mesures effectuées sur un couvert de luzerne en région parisienne et sa sensibilité vis-à-vis des différents paramètres est examinée.
\end{abstract}

Mots clés additionnels : Modèle mathématique, couvert végétal. temperature.

We have developed an analog model to calculate the evapotranspiration rate of a canopy from measurements of stomatal resistance and leaf temperature. The model was based on the division of the stand into different horizontal layers. Each layer was characterized by two temperatures ${ } r_{i}$ and $T s_{i}$ and two elementary resistances $\mathrm{ra}_{\mathrm{i}}$ and $\mathrm{re}_{\mathrm{i}}$ (fig. 2 and 3). $\mathrm{Tr}_{\mathrm{i}}$ represented the dew point temperature of the air in layer $\mathrm{i}$ and $\mathrm{Ts}_{\mathrm{i}}$ the mean temperature of the leaves; $\mathrm{ra}_{\mathrm{i}}$ characterized the diffusion resistance to water vapour across layer $\mathrm{i}$ and $\mathrm{re}_{\mathrm{i}}$ the diffusion resistance inside the layer (from the stomatal cavities to the top of the layer) (fig. 1). The model led to a mathematical expression of the global evaporation flux as a function of the dew point temperature of the air at the top of the canopy $\operatorname{Tr}\left(z_{h}\right)$, the leaf surface temperatures $T s_{i}$ and the elementary resistances $r a_{i}$ and $r e_{i}$. It was tested with data obtained from a lucerne crop in the Paris area and its sensitivity to the different parameters was examined.

Additional key words : Mathematical model, crop canopy.

\section{INTRODUCTION}

$\mathrm{Si}$ on veut calculer à partir d'un échantillon de valeurs ponctuelles de résistance stomatique l'évapotranspiration globale du couvert végétal, la méthode classique consiste à utiliser l'équation de PENMANMONTEITH et à déterminer la résistance du couvert $R v$ qui apparaît dans l'équation à partir de l'échantillon considéré.

Rappelons que l'équation de PenMan-Monteith peut se mettre sous la forme suivante :

$$
\mathrm{ETR}=\frac{\mathrm{P}^{\prime} \cdot\left(\mathrm{Rn}+\phi_{0}\right)+\gamma \cdot \mathrm{Ea}}{\mathrm{P}^{\prime}+\gamma(\mathrm{Rv}+\mathrm{Ra}) / \mathrm{Ra}} .
$$

La résistance Rv traduit le frein qu'oppose le couvert végétal à la diffusion de la vapeur d'eau, ce frein se définissant par rapport au cas d'une nappe d'eau libre. Pour MonteITH (1965), Rv est liée essentiellement au degré d'ouverture des stomates $(\mathbf{R v}=\mathbf{R s})$. 
TABLEAU 1

Liste des symboles.

List of symbols.

\begin{tabular}{|c|c|}
\hline Ea & pouvoir évaporant de l'air : $\mathrm{Ea}=\mathrm{k} \cdot\left(\mathrm{T}\left(\mathrm{z}_{\mathrm{T}}\right)-\operatorname{Tr}\left(\mathrm{z}_{\mathrm{r}}\right)\right) / \mathrm{Ra}$ \\
\hline$c_{p}$ & chaleur massique de l'air \\
\hline ETR & évapotranspiration réelle du couvert végétal \\
\hline $\mathrm{h}$ & coefficient d'échange des surfaces foliaires \\
\hline i & indice rapportant la grandeur correspondante à la strate $\mathrm{i}$ \\
\hline $\mathrm{K}$ & diffusivité turbulente \\
\hline $\mathrm{k}=$ & $\rho c_{p} P^{\prime} \gamma$ \\
\hline LAI & indice de surface foliaire \\
\hline $\mathrm{P}^{\prime}$ & pente de la courbe de pression de vapeur saturante \\
\hline $\mathrm{Ra}$ & résistance aérodynamique au-dessus du couvert \\
\hline Rs & résistance stomatique globale du couvert ou d'une strate \\
\hline Rv & résistance globale du couvert végétal \\
\hline rs & $\begin{array}{l}\text { résistance stomatique moyenne de la face supérieure des } \\
\text { feuilles d'une strate }\end{array}$ \\
\hline ri & $\begin{array}{l}\text { résistance stomatique moyenne de la face inférieure des } \\
\text { feuilles d'une strate }\end{array}$ \\
\hline ra & résistance aérodynamique d'une strate de végétation \\
\hline re & résistance équivalente d'une strate \\
\hline $\mathrm{Rn}$ & rayonnement net du couvert \\
\hline $\mathbf{T}$ & température de l'air \\
\hline Ts & température de surface des feuilles \\
\hline $\operatorname{Tr}$ & température de rosée de l’air \\
\hline $\mathrm{U}$ & vitesse du vent \\
\hline $\mathrm{z}_{\mathrm{r}}$ & niveau de référence climatique \\
\hline$z_{0}$ & hauteur de rugosité \\
\hline $\mathrm{z}_{\mathrm{h}}$ & hauteur du couvert végẹ́tal \\
\hline$\gamma$ & constante psychrométrique \\
\hline$\varphi$ & flux élémentaire de vapeur d'eau au niveau d'une strate \\
\hline$\phi$ & flux global de vapeur d'eau \\
\hline$\rho$ & masse volumique de l'air \\
\hline$\phi_{0}$ & flux conductif de chaleur à la surface du sol \\
\hline
\end{tabular}

PERRIER (1975), qui reprit plus tard avec quelques modifications le même genre d'équation, considère $\mathrm{Rv}$ comme la somme de 2 résistances : l'une Ro qui traduit le frein dû à la structure du couvert et l'autre Rs représentant la résistance proprement stomatique au sens de MONTEITH $(\mathbf{R v}=\mathrm{Ro}+\mathrm{Rs})$.

Pour relier Rs aux mesures ponctuelles de résistance stomatique, MONTEITH (1973) a proposé la méthode suivante. Il divise le couvert en $\mathbf{n}$ strates parallèles, chacune ayant une résistance $R s_{\mathrm{i}}$. La résistance globale Rs représente la résistance équivalente à la série des résistances $\mathbf{R s}_{\mathrm{i}}$ mises en parallèle :

$$
1 / \mathrm{Rs}=\sum_{\mathbf{i}}\left(1 / \mathrm{Rs}_{\mathrm{i}}\right) .
$$

Chaque résistance $\mathrm{Rs}_{\mathrm{i}}$ est fonction de l'indice foliaire $\mathrm{LAI}_{\mathrm{i}}$ et de la résistance stomatique ponctuelle moyenne $\overline{\mathrm{r}}_{\mathrm{i}}$ de la strate considérée :

$$
\mathrm{Rs}_{\mathrm{i}}=\overline{\mathrm{r}}_{\mathrm{i}} / \mathrm{LAI}_{\mathrm{i}} \text {. }
$$

La résistance $\overline{\mathrm{r}_{\mathrm{i}}}$ étant elle-même calculée à partir des résistances stomatiques moyennes des faces supérieu-

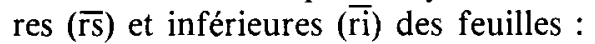

$$
1 / \overline{\mathrm{r}}_{\mathrm{i}}=1 / \overline{\mathrm{r}}_{\mathrm{i}}+1 / \overline{\mathrm{r}}_{\mathrm{i}} .
$$

Ce schéma de calcul constitue un moyennage des conductances stomatiques des différentes strates, chaque conductance étant pondérée par la surface foliaire de la strate correspondante. Mais il est bon de souligner que ce schéma repose sur un certain nombre d'hypothèses simplificatrices. D'abord la part du sol dans le processus d'évapotranspiration est négligée.
Ensuite, pour écrire l'équation (2), on est obligé de supposer que toutes les strates sont au même « potentiel ", c'est-à-dire que toutes les feuilles ont la même température (puisque à l'intérieur des cavités stomatiques la pression de vapeur est supposée être égale à la pression saturante). Enfin les équations (3) et (4) négligent les résistances de type aérodynamique que rencontre la vapeur dans son processus de diffusion de la surface des feuilles jusqu'au sommet du couvert.

Compte tenu de ces considérations, nous avons cherché à mettre au point une méthode de calcul plus cohérente et plus complète qui permette d'éliminer une partie des hypothèses du modèle de MONTEITH. C'est le propos de cet article de présenter les résultats obtenus.

\section{DESCRIPTION DU MODÈLE}

Nous considérerons un couvert végétal homogène et horizontal que nous diviserons en un certain nombre de strates ou tranches parallèles. Chacune sera caractérisée par un indice de surface foliaire partielle $\mathrm{LAI}_{\mathrm{i}}$, une température moyenne de surface foliaire $\mathrm{Ts}_{\mathrm{j}}$ et 2 résistances stomatiques moyennes, une pour la face supérieure des feuilles $\mathrm{rs}_{\mathrm{i}}$ et l'autre pour la face inférieure $\mathrm{ri}_{\mathrm{i}}$. L'air au niveau de la strate i sera caractérisé par une température de rosée $\operatorname{Tr}_{\mathrm{i}}$ et une vitesse de vent $\mathrm{U}_{\mathrm{i}}$ dont dépend le coefficient d'échange $h_{\mathrm{i}}$. Chaque strate de végétation est en quelque sorte assimilée à une grande feuille de surface $\mathrm{LAI}_{\mathrm{i}}$ possédant les caractéristiques moyennes de la strate considérée.

Le modèle est basé sur une analogie électrique où le flux de vapeur d'eau remplace l'intensité du courant et la température de rosée le potentiel électrique. On peut considérer que la vapeur d'eau dans les cavités stomatiques est saturante à la température $\mathrm{Ts}_{\mathrm{i}}$ et qu'elle emprunte, dans son processus de diffusion, 2 voies en parallèle, correspondant aux 2 faces des feuilles. Chaque voie se compose de 2 résistances en série, la résistance stomatique et celle de la couche limite de la feuille. Le schéma électrique équivalant à ce processus de diffusion est donné par la figure 1 . La résistance équivalente, pour chaque strate de végétation, est la suivante :

$$
\mathrm{re}_{\mathrm{i}}=\left(\frac{1}{1 / \mathrm{h}_{\mathrm{i}}+\mathrm{rs}_{\mathrm{i}}}+\frac{1}{1 / \mathrm{h}_{\mathrm{i}}+\mathrm{ri}_{\mathrm{i}}}\right)^{-1} / \mathrm{LAI}_{\mathrm{i}} .
$$

Le flux élémentaire de vapeur d'eau qui lui correspond, s'écrivant :

$$
\varphi_{\mathrm{i}}=\mathrm{k} \cdot\left(\mathrm{Ts}_{\mathrm{i}}-\operatorname{Tr}_{\mathrm{i}}\right) / \mathrm{re}_{\mathrm{i}}
$$

$\operatorname{avec} \mathrm{k}=\rho \cdot \mathrm{c}_{\mathrm{p}} \cdot \mathbf{P}^{\prime} / \gamma$.

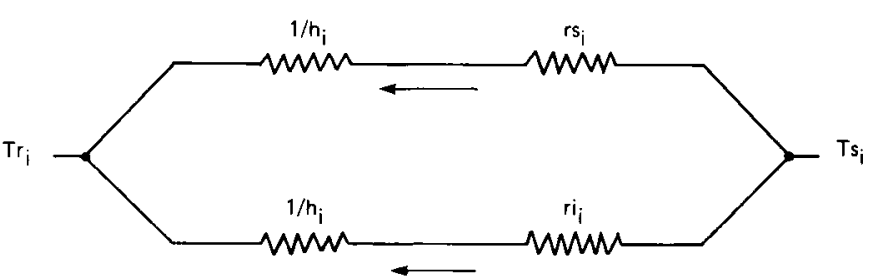

Figure 1.

Analogie électrique correspondant au processus de diffusion de la vapeur d'eau à l'intérieur d'une strate de végétation.

Electrical analogy showing the water vapour diffusion process inside a vegetation layer. 
Le flux élémentaire $\varphi_{\mathrm{i}}$ qui provient de la strate i va se mélanger au flux général qui provient des tranches de végétation inférieures et qu'on notera $\phi_{i+1}$. Ce flux $\phi_{i+1}$, pour traverser la tranche de végétation $\mathrm{i}$, va rencontrer une résistance aérodynamique $\mathrm{ra}_{\mathrm{i}}$. La figure 2 présente le schéma électrique qui visualise la jonction des flux $\varphi_{\mathrm{i}}$ et $\phi_{\mathrm{i}+1}$ et le nouveau flux $\phi_{\mathrm{i}}$ qui en résulte. En toute rigueur, le point de jonction entre le flux élémentaire $\varphi_{\mathrm{i}}$ et le flux général $\phi_{\mathrm{i}+1}$, de potentiel $\mathrm{Tr}_{\mathrm{i}}$, devrait se trouver au milieu de la résistance $\mathrm{ra}_{\mathrm{i}}$. Mais pour simplifier, nous le mettrons audessus, considérant que l'erreur introduite est négligeable.

Le schéma électrique équivalant au couvert pris dans son ensemble et divisé en $n$ strates se présente comme une succession de $\mathrm{n}$ schémas élémentaires du type donné par la figure 2. Remarquons que ce schéma général (fig. 3) permet de tenir compte de l'évaporation au niveau du sol. En effet, on peut très bien assimiler la couche de sol évaporante à une strate de végétation de température caractéristique $\mathrm{Ts}_{\mathrm{n}}$ (température de surface du sol) et de résistance équivalente $\mathrm{re}_{\mathrm{n}}=1 / \mathrm{h}_{\mathrm{n}}+\mathrm{r}_{\mathrm{s}}, \mathrm{r}_{\mathrm{s}}$ représentant la résistance à la diffusion de la couche superficielle de sol et $h_{n}$ le coefficient d'échange correspondant (PERRIER, 1975). Le flux global de vapeur d'eau $\phi$, ou évapotranspiration du couvert, représente la somme des flux élémentaires $\varphi_{\mathrm{i}}$ existant au niveau de chaque tranche du couvert :

$$
\phi=\sum_{i=1}^{n} \varphi_{i} .
$$

Soit, en tenant compte de l'équation (6) :

$$
\phi=\mathrm{k} \cdot\left(\sum_{\mathrm{i}=1}^{\mathrm{n}} \mathrm{Ts}_{\mathrm{i}} / \mathrm{re}_{\mathrm{i}}-\sum_{\mathrm{i}=1}^{\mathrm{n}} \operatorname{Tr}_{\mathrm{i}} / \mathrm{re}_{\mathrm{i}}\right) .
$$

Les grandeurs $T s_{i}$ et $\mathrm{re}_{\mathrm{i}}$ sont supposées connues ou accessibles à partir de l'expérimentation. Le problème consiste alors à exprimer les températures de rosée $\mathrm{Tr}_{\mathrm{i}}$ aux nœuds du réseau, a priori inconnues, en fonction de paramètres qui eux sont connus.

En annexe nous développons les calculs qui permettent de déterminer $\operatorname{Tr}_{\mathrm{i}}$ et qui conduisent finalement à l'expression suivante du flux global de vapeur d'eau :

$$
\phi=k \cdot \frac{\left(-\sum_{i=1}^{n} E_{i} \cdot \operatorname{Ts} / A\right)-\operatorname{Tr}\left(z_{h}\right)}{\left(1+r a_{1} \cdot B\right) / A} .
$$

Les coefficients $A, B$ et $E_{i}$ s'expriment uniquement en fonction des résistances élémentaires $\mathrm{ra}_{\mathrm{i}}$ et $\mathrm{re}_{\mathrm{i}}$ et sont homogènes à l'inverse d'une résistance.

\section{CALCUL PRATIQUE DE L'ÉVAPOTRANSPIRATION}

Les étapes de calcul de l'évapotranspiration à partir des mesures ponctuelles de résistance stomatique sont les suivantes. Le couvert ayant été divisé en n strates parallèles, on détermine pour chaque strate i la surface foliaire partielle $\mathrm{LAI}_{\mathrm{i}}$ et on effectue une série de mesures au poromètre à diffusion sur des plantes

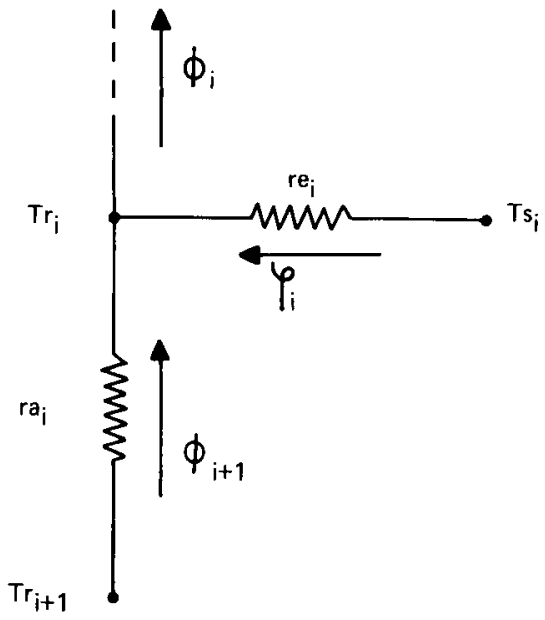

Figure 2.

Analogie électrique correspondant au processus de diffusion de la vapeur d'eau au travers d'une strate de végétation.

Electrical analogy showing the water vapour diffusion process across a vegetation layer.

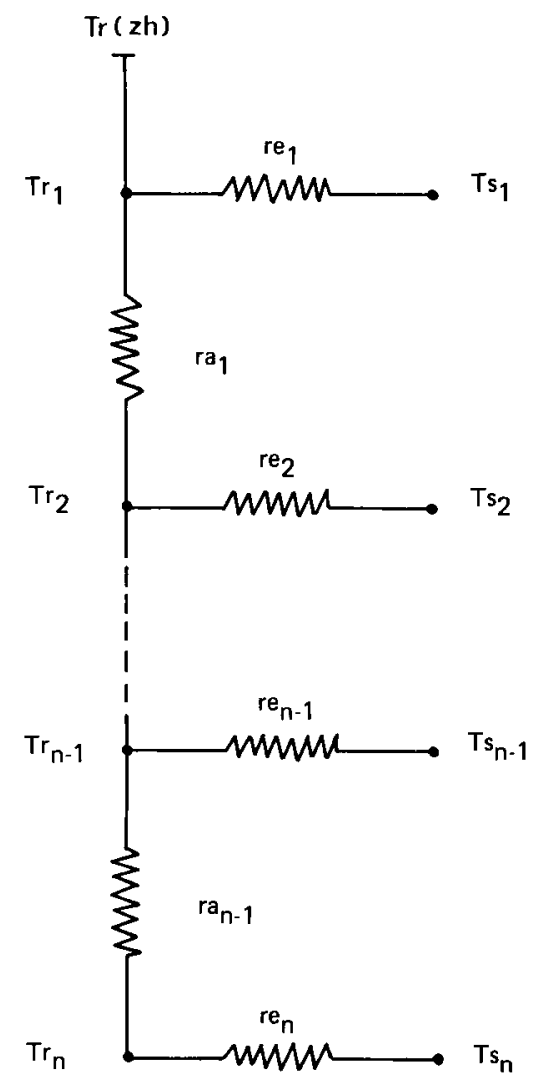

Figure 3.

Analogie électrique correspondant au processus de diffusion de la vapeur d'eau à l'intérieur du couvert.

Electrical analogy showing the water vapour diffusion process inside the whole crop.

choisies au hasard, en nombre égal sur les faces supérieures et inférieures des feuilles, le poromètre mesurant la résistance stomatique et la température foliaire (KATERJI \& OULID-AISSA, 1983). On calcule alors, pour chaque strate, la température moyenne de surface des feuilles $\overline{\mathrm{Ts}}_{\mathrm{i}}$ et les résistances stomatiques 
moyennes des faces supérieures $\overline{\mathrm{rs}}_{\mathrm{i}}$ et inférieures $\overline{\mathrm{r}}_{\mathrm{i}}$ :

$$
\begin{gathered}
\overline{\overline{T s}_{i}}=\frac{1}{m} \cdot \sum_{j=1}^{m}\left(\mathrm{Ts}_{\mathrm{i}}\right)_{j} \\
\overline{\overline{\mathrm{rs}_{i}}}=\frac{1}{\mathrm{~m}} \cdot \sum_{\mathrm{j}=1}^{\mathrm{m}} \frac{1}{\left(\mathrm{rs}_{\mathrm{i}}\right)_{\mathrm{j}}} \\
\frac{1}{\overline{\overline{\mathrm{ri}}}}=\frac{1}{\mathrm{~m}} \cdot \sum_{\mathrm{j}=1}^{\mathrm{m}} \frac{1}{\left(\mathrm{ri}_{\mathrm{i}}\right)_{j}} .
\end{gathered}
$$

La résistance stomatique représentant l'inverse d'une conductance c'est la moyenne harmonique qui doit être utilisée au lieu de la moyenne arithmétique.

L'étape suivante du calcul consiste à déterminer, pour chaque strate de végétation, les résistances élémentaires $\mathrm{re}_{\mathrm{i}}$ et $\mathrm{ra}_{\mathrm{i}}$. La détermination de la résistance $\mathrm{re}_{i}$ à partir de la formule (5) nécessite, outre la connaissance de $L A I_{i}$, $r s_{i}$ et $r i_{i}$, celle du coefficient d'échange $h_{i}$ des feuilles. Ce coefficient d'échange est une fonction de la vitesse du vent qui se met généralement sous la forme suivante :

$$
\mathrm{h}_{\mathrm{i}}=\mathrm{h}_{0} \cdot \mathrm{U}_{\mathrm{i}}^{\mathrm{a}}
$$

où $h_{0}$ peut être considéré comme une constante en première approximation. L'exposant a varie suivant les auteurs entre 0,5 et 1 . PERRIER (1968) propose $a=0,8$. Dans ce cas, $h_{0}$ est voisin de 0,02 (pour l'une des faces de la feuille). $U_{\mathrm{i}}$ représente la vitesse du vent au niveau de la strate $i$. La résistance $\mathrm{ra}_{\mathrm{i}}$, quant à elle, s'exprime en fonction de l'épaisseur $\Delta z_{i}$ de la strate $i$ et de la diffusivité turbulente $K_{i}$ au niveau de cette strate de la manière suivante :

$$
\mathrm{ra}_{\mathrm{i}}=\Delta \mathrm{z}_{\mathrm{i}} / \mathrm{K}_{\mathrm{i}} .
$$

Pour connaître la vitesse du vent $\mathrm{U}_{\mathrm{i}}$ et la diffusivité turbulente $K_{i}$ au niveau de chaque strate on peut utiliser les profils théoriques proposés par PERRIER (1967, 1976) qui lient la vitesse du vent et la diffusivité à un niveau $\mathrm{z}$ au sein du couvert à la vitesse du vent $\mathrm{U}(\mathrm{zh})$ au sommet du couvert :

$$
\begin{aligned}
& U(z)=U(z h) \cdot \exp \cdot\left(-B_{0} \cdot F(z)\right) \\
& K(z)=A_{0} \cdot B_{0} \cdot U(z) / f(z)
\end{aligned}
$$

$f(z)$ est la fonction densité de surface foliaire et $F(z)$ définit la surface foliaire cumulée à partir du sommet du couvert (PERRIER, 1975). $A_{0}$ et $B_{0}$ sont 2 coefficients théoriques voisins respectivement de 0,4 et 0,6 . Compte tenu de la discrétisation du couvert, $\mathrm{U}_{\mathrm{i}}$ et $\mathrm{K}_{\mathrm{i}}$ s'écrivent respectivement :

$$
\begin{aligned}
& \mathrm{U}_{\mathrm{i}}=\mathrm{U}(\mathrm{zh}) \cdot \exp \cdot\left(-\mathrm{B}_{0} \cdot \sum_{\mathrm{j}=1}^{\mathrm{i}-1} \mathrm{LAI}_{\mathrm{j}}\right) \\
& \mathrm{K}_{\mathrm{i}}=\mathrm{A}_{0} \cdot \mathrm{B}_{0} \cdot \mathrm{U}_{\mathrm{i}} /\left(\mathrm{LAI}_{\mathrm{i}} / \Delta \mathrm{z}_{\mathrm{i}}\right)
\end{aligned}
$$

$\sum \mathrm{LAI}_{\mathrm{ji}}$ représentant la surface foliaire cumulée $\mathrm{du}$ sommet jusqu'à la strate $\mathrm{i}$ exclue et $\Delta \mathrm{z}_{\mathrm{i}}$ l'épaisseur de la strate $\mathrm{i}$.

Une fois déterminées les résistances $r a_{i}$ et $r e_{i}$ pour chaque strate de végétation, il est facile de calculer les coefficients $a_{i}, b_{i}$ et $c_{i}$ (formules (d) de l'annexe). L'algorithme (g), auquel s'ajoute le cas supplémentaire $\varepsilon_{1}^{i}=-1$, permet alors de déterminer les coef- ficients $A, B$ et $E_{i}$ qui entrent dans l'expression du flux global de vapeur d'eau (9). Pratiquement il est plus facile d'utiliser directement l'algorithme avec les valeurs numériques, et ceci au moyen d'un petit programme de calcul, que d'essayer de reconstituer la formulation algébrique du flux gobal $\phi$ en fonction des résistances élémentaires $\mathrm{re}_{\mathrm{i}}$ et $\mathrm{ra}_{\mathrm{i}}$. Car très rapidement $(n \geq 3)$ les expressions algébriques deviennent compliquées.

\section{VÉRIFICATION EXPÉRIMENTALE}

Des mesures ont été réalisées à la Station de Bioclimatologie de Versailles sur des couverts de luzerne et de pomme de terre. Dans chaque cas la culture est divisée en 2 ou 3 strates de $20-30 \mathrm{~cm}$ chacune selon la croissance. Avec un pas de temps d'une heure, une vingtaine de mesures de résistance stomatique et de température foliaire sont réalisées dans chaque strate, à égalité sur les faces supérieures et inférieures des feuilles. En même temps, des mesures d'évapotranspiration de la culture considérée sont effectuées avec un système BEARN (PERRIER et al., 1975) dont le principe repose sur la méthode du rapport de Bowen.

Dans le tableau 2 nous présentons des résultats obtenus sur pomme de terre lors d'une journée de mesure (le 29 juillet 1983), le couvert ayant une hauteur moyenne $z_{h}=56 \mathrm{~cm}$. Ce tableau montre la gamme de variation des grandeurs mesurées. En ce qui concerne les résistances stomatiques de la strate inférieure, nous avons adopté systématiquement la valeur moyenne de $1500 \mathrm{s.m} \mathrm{m}^{-1}$ sans effectuer de mesures. Ceci s'explique par le fait que, lorsque le couvert dépasse $50 \mathrm{~cm}$ de hauteur, les stomates de la strate inférieure sont pratiquement fermés et exigent un temps de mesure très long (4-5 $\mathrm{mn}$ par mesure) incompatible avec le pas de temps d'une heure imparti à l'ensemble des mesures. Mais la valeur moyenne adoptée est celle généralement obtenue dans cette couche lorsque la hauteur du couvert dépasse $50 \mathrm{~cm}$.

Le modèle proposé a été confronté à l'expérience de la manière suivante. Les valeurs d'évapotranspiration déduites des mesures de résistance stomatique et de température foliaire au moyen des équations du modèle sont comparées aux valeurs données par le système BEARN. La figure 4 montre les résultats obtenus sur un couvert de luzerne bien couvrant, dont l'évaporation du sol peut être considérée comme négligeable en première approximation. La comparaison porte sur 90 couples de valeurs (moyennes horaires). La droite d'ajustement par la méthode des moindres carrés s'écrit :

$$
\mathrm{ETC}=50+0,88 . \mathrm{ETB} \quad\left(\mathrm{W} \cdot \mathrm{m}^{-2}\right)
$$

avec un coefficient de corrélation de 0,82 . Et le coefficient $\mathrm{k}$ moyen de la relation ETC $=\mathrm{k}$. ETB, qui permet de mieux apprécier la précision de l'estimation, vaut 1,04 . La dispersion relativement importante des résultats s'explique aisément si on tient compte, d'une part, de la faible précision avec laquelle sont effectuées les mesures d'ordre biologique (résistance stomatique, température de surface foliaire) et des erreurs d'échantillonnage qui y sont liées et, d'autre part, du fait que les valeurs données par le BEARN ne représentent pas une référence absolue mais sont, elles-aussi, 
TABLEAU 2

Evolution au cours d'une journée des résistances stomatiques et des températures foliaires d'un couvert de pomme de terre $\left(z_{h}=56 \mathrm{~cm}, L A I=4,7\right)$.

Daily variation in stomatal resistance and leaf temperature observed in a potato crop $\left(z_{h}=56 \mathrm{~cm}, L A I=4.7\right)$.

\begin{tabular}{|c|c|c|c|c|c|c|c|c|c|c|c|c|}
\hline & & & $\begin{array}{r}9 \text { h } 00 \\
10 \text { h } 00 \\
\end{array}$ & $\begin{array}{l}10 \text { h } 00 \\
11 \text { h } 00\end{array}$ & $\begin{array}{l}12 \text { h } 00 \\
13 \text { h } 00\end{array}$ & $\begin{array}{l}13 \text { h } 00 \\
14 \text { h } 00\end{array}$ & $\begin{array}{l}14 \text { h } 00 \\
15 \text { h } 00\end{array}$ & $\begin{array}{l}15 \text { h } 00 \\
16 \text { h } 00\end{array}$ & $\begin{array}{l}16 \text { h } 00 \\
17 \text { h } 00\end{array}$ & $\begin{array}{l}17 \text { h } 00 \\
18 \text { h } 00\end{array}$ & $\begin{array}{l}18 \text { h } 00 \\
19 \text { h } 00\end{array}$ & Unité \\
\hline \multirow{4}{*}{ 离 } & \multirow{2}{*}{$\begin{array}{l}\text { face } \\
\text { sup. }\end{array}$} & rs & 120 & 203 & 152 & 180 & 154 & 185 & 319 & 414 & 632 & $\mathrm{~s} \cdot \mathrm{m}^{-1}$ \\
\hline & & Ts & 25,5 & 25,8 & 26,5 & 29,3 & 29,8 & 30,7 & 30,0 & 28,9 & 27,6 & ${ }^{\circ} \mathrm{C}$ \\
\hline & \multirow{2}{*}{$\begin{array}{l}\text { face } \\
\text { inf. }\end{array}$} & $\mathrm{ri}$ & 117 & 127 & 100 & 143 & 133 & 173 & 229 & 336 & 678 & s. $\mathrm{m}^{-1}$ \\
\hline & & Ts & 26,9 & 27,2 & 29,5 & 29,9 & 30,3 & 31,2 & 30,0 & 29,6 & 26,6 & ${ }^{\circ} \mathrm{C}$ \\
\hline \multirow{4}{*}{ 莺 } & \multirow{2}{*}{$\begin{array}{l}\text { face } \\
\text { sup. }\end{array}$} & rs & 230 & 527 & 446 & 797 & 445 & 944 & 443 & 1138 & 1200 & s. $\mathrm{m}^{-1}$ \\
\hline & & Ts & 26,4 & 26,9 & 29,3 & 29,3 & 29,9 & 30,7 & 30,1 & 28,4 & 26,0 & ${ }^{\circ} \mathrm{C}$ \\
\hline & \multirow{2}{*}{$\begin{array}{l}\text { face } \\
\text { inf. }\end{array}$} & $\mathrm{ri}$ & 399 & 415 & 462 & 527 & 450 & 1149 & 369 & 1127 & 1200 & $\mathrm{~s} \cdot \mathrm{m}^{-1}$ \\
\hline & & Ts & 24,7 & 26,7 & 29,4 & 28,7 & 29,5 & 30,4 & 28,3 & 28,0 & 25,2 & ${ }^{\circ} \mathrm{C}$ \\
\hline \multirow{4}{*}{ 莺 } & \multirow{2}{*}{$\begin{array}{l}\text { face } \\
\text { sup. }\end{array}$} & rs & 1500 & 1500 & 1500 & 1500 & 1500 & 1500 & 1500 & 1500 & 1500 & s.m ${ }^{-1}$ \\
\hline & & Ts & 26,4 & 26,9 & 29,3 & 29,3 & 29,9 & 30,7 & 30,0 & 28,4 & 25,2 & ${ }^{\circ} \mathrm{C}$ \\
\hline & \multirow{2}{*}{$\begin{array}{l}\text { face } \\
\text { inf. }\end{array}$} & ri & 1500 & 1500 & 1500 & 1500 & 1500 & 1500 & 1500 & 1500 & 1500 & s. $\mathrm{m}^{-1}$ \\
\hline & & Ts & 24,7 & 26,7 & 29,4 & 28,7 & 29,5 & 30,4 & 28,3 & 28,0 & 25,2 & ${ }^{\circ} \mathrm{C}$ \\
\hline & \multicolumn{2}{|l|}{$U\left(z_{T}\right)$} & 2,9 & 2,4 & 1,9 & 1,8 & 1,9 & 2,0 & 2,2 & 2,0 & 1,3 & $\mathrm{~m} \cdot \mathrm{s}^{-1}$ \\
\hline & \multicolumn{2}{|l|}{$I\left(z_{T}\right)$} & 21,4 & 22,9 & 25,6 & 25,4 & 25,7 & 25,9 & 26,6 & 26,6 & 25,6 & ${ }^{\circ} \mathrm{C}$ \\
\hline & \multicolumn{2}{|c|}{$\mathrm{Rn}+\phi_{0}$} & 428 & 510 & 546 & 453 & 433 & 356 & 254 & 185 & 41 & W. $\mathrm{m}^{-2}$ \\
\hline & \multicolumn{2}{|c|}{$\phi$} & 411 & 493 & 535 & 451 & 420 & 349 & 260 & 185 & 41 & W. $\mathrm{m}^{-2}$ \\
\hline
\end{tabular}

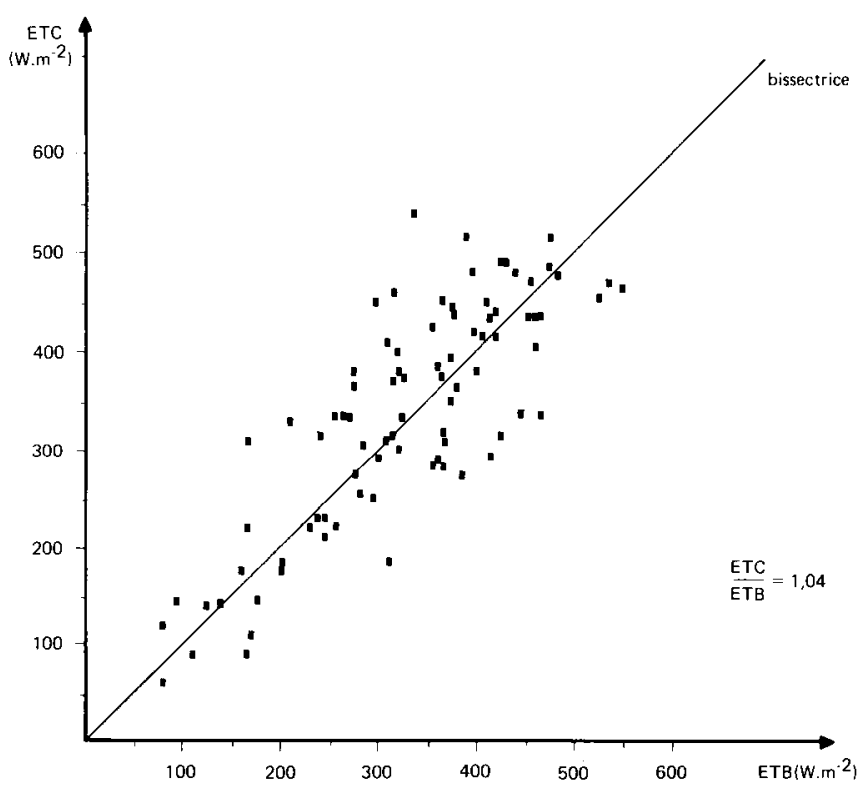

Figure 4.

Comparaison entre valeurs horaires de l'évapotranspiration calculées à partir du modèle (ETC) et mesurées par le BEARN (ETB). Comparison between hourly values of actual evapotranspiration rates calculated from the model (ETC) and measured with the BEARN system (ETB).

entachées d'erreurs. Globalement il est donc légitime de penser que la confrontation expérimentale est positive.
Pour étudier la sensibilité du modèle à une variation des paramètres nous avons procédé de la manière suivante. Le tableau 3 donne un profil-type observé sur une culture de luzerne (résistance stomatique, température foliaire et indice foliaire). Chaque valeur est accompagnée de l'écart-type dû à l'échantillonnage. A partir de ces valeurs, nous avons calculé pour chaque paramètre la variation du flux de vapeur correspondant à une variation du paramètre égale à son intervalle de confiance ( \pm écart-type). Les résultats apparaissent dans le tableau 4 . Ils mettent en évidence que le modèle est très peu sensible à une erreur sur les valeurs de résistance stomatique : une erreur de 25 p. 100 sur les résistances de la strate supérieure ne provoque qu'une erreur de 10 p. 100 sur la valeur du flux global d'évapotranspiration. Pour les strates inférieures, la sensibilité est encore moindre : une variation de 35 p. 100 de la résistance correspond à une variation de seulement 5 p. 100 de l'évapotranspiration. Le modèle est par contre beaucoup plus sensible aux erreurs sur la température foliaire puisque une variation de $0,8^{\circ} \mathrm{C}$ sur Ts provoque une variation de 8 p. 100 sur le flux global. Quant à la sensibilité du modèle vis-à-vis de l'indice foliaire LAI, elle est relativement faible : une variation de 20 p. 100 sur LAI correspond à une variation de seulement 10 p. 100 sur le flux de vapeur. De cette étude de sensibilité, il ressort principalement que la variabilité naturellement importante de la résistance stomatique (KATERJI et al., 1983) influe peu sur la valeur du flux d'évapotranspiration. 
TABLEAU 3

Profils de résistance stomatique, température foliaire et indice foliaire observés sur un couvert de luzerne (moyenne et écart-type de chaque échantillon).

Profiles of stomatal resistance, leaf temperature and leaf area index observed on a lucerne crop (mean value and standard deviation of each sample).

\begin{tabular}{|c|c|c|c|c|c|c|c|}
\hline & \multicolumn{2}{|c|}{ Strate supérieure } & \multicolumn{2}{|c|}{ Strate intermédiaire } & \multicolumn{2}{|c|}{ Strate inférieure } & \multirow[t]{2}{*}{ Unité } \\
\hline & $\begin{array}{l}\text { face } \\
\text { sup. }\end{array}$ & $\begin{array}{l}\text { face } \\
\text { inf. }\end{array}$ & $\begin{array}{l}\text { face } \\
\text { sup. }\end{array}$ & $\begin{array}{l}\text { face } \\
\text { inf. }\end{array}$ & $\begin{array}{l}\text { face } \\
\text { sup. }\end{array}$ & $\begin{array}{l}\text { face } \\
\text { inf. }\end{array}$ & \\
\hline Résistance stomatique & $117 \pm 29$ & $115 \pm 29$ & $199 \pm 70$ & $559 \pm 196$ & $1044 \pm 365$ & $1200 \pm 420$ & s. m-1 \\
\hline Température de surface & $19,6 \pm 0,9$ & $21,1 \pm 0,4$ & $20,0 \pm 1,0$ & $18,1 \pm 1,1$ & $18,5 \pm 0,6$ & $18,5 \pm 0,4$ & ${ }^{\circ} \mathrm{C}$ \\
\hline LAl & \multicolumn{2}{|c|}{$1,75 \pm 0,35$} & \multicolumn{2}{|c|}{$2,10 \pm 0,42$} & \multicolumn{2}{|c|}{$0,85 \pm 0,17$} & $\mathrm{~m}^{2}$ \\
\hline
\end{tabular}

$\mathrm{z}_{\mathrm{h}}=0,62 \mathrm{~m}, \quad \mathrm{U}\left(\mathrm{z}_{\mathrm{r}}\right)=2,8 \mathrm{~m} \cdot \mathrm{s}^{-1}, \quad \mathrm{~T}\left(\mathrm{z}_{\mathrm{r}}\right)=13,7{ }^{\circ} \mathrm{C}, \quad \operatorname{Tr}\left(\mathrm{z}_{\mathrm{r}}\right)=5,4^{\circ} \mathrm{C}$.

TABLEAU 4

Sensibilité du modèle à la variation des paramètres.

Variation in evapotranspiration flux as a function of variation in each parameter.

\begin{tabular}{|c|c|c|c|}
\hline Paramètre & $\begin{array}{l}\text { Valeur } \\
\text { moyenne }\end{array}$ & $\begin{array}{l}\text { Variation du } \\
\text { paramètre }\end{array}$ & $\begin{array}{c}\text { Variation } \\
\text { correspondante } \\
\text { du flux }\end{array}$ \\
\hline $\begin{array}{l}\text { r(strate sup.) } \\
\text { s. } \mathrm{m}^{-1}\end{array}$ & 58 & $\pm 15 \quad( \pm 25 \%)$ & $\pm 10 \%$ \\
\hline $\begin{array}{l}\mathrm{r} \text { (strate int.) } \\
\text { s. } \mathrm{m}^{-1}\end{array}$ & 147 & $\pm 51 \quad( \pm 35 \%)$ & $\pm 5 \%$ \\
\hline $\begin{array}{l}\text { r(strate inf.) } \\
\text { s. } \mathrm{m}^{-1}\end{array}$ & 558 & $\pm 195( \pm 35 \%)$ & $\pm 5 \%$ \\
\hline $\mathrm{T}_{\mathrm{s}}\left({ }^{\circ} \mathrm{C}\right)$ & 20 & $\pm 0,8$ & $\pm 8 \%$ \\
\hline LAI & 4,7 & $\pm 0,94( \pm 20 \%)$ & $\pm 10 \%$ \\
\hline
\end{tabular}

\section{CONCLUSION}

Si nous reprenons les principales hypothèses citées en introduction sur lesquelles repose la méthode de MONTEITH, nous pouvons constater que le modèle présenté les élimine presque toutes. En effet nous avons vu que le modèle permet de prendre en considération l'évaporation du sol si on connaît expérimentalement la résistance à la diffusion et le coefficient d'échange de la couche superficielle de sol. Le modèle ne suppose pas une température uniforme pour tout le couvert mais considère que chaque strate est caractérisée par une température de surface foliaire $\mathrm{Ts}_{\mathrm{i}}$. Enfin les résistances à la diffusion au sein du couvert, de type aérodynamique, sont prises en considération à travers les coefficients d'échange des feuilles $h_{i}$ et les résistances élémentaires $r a_{i}$.

Mais le modèle d'évapotranspiration présenté ne constitue pas seulement une amélioration de la méthode de MONTEITH. Basé sur une analogie électrique qui assimile le couvert végétal, dans son processus de diffusion de la vapeur d'eau, à un réseau de résistances, il devrait pouvoir servir d'outil d'analyse pour mieux comprendre le déterminisme de l'évapotranspiration en fonction des caractéristiques biologiques et architecturales du couvert.

Reçu le 9 novembre 1983. Accepté le 10 décembre 1984.

\section{RÉFÉRENCES BIBLIOGRAPHIQUES}

Katerji N., Perrier A., Oulid-Aissa A. K., 1983. Exploration au champ et interprétation de la variation horizontale et verticale de la résistance stomatique : cas d'une culture de luzerne. Agronomie, 3 (9), 847-856.

Katerii N., Oulid-Aissa A. K., 1983. Etude et amélioration de la fiabilité d'un poromètre à diffusion utilisé en milieu naturel. Agronomie, 3 (9) 931-936.

Monteith J. L., 1973. Principles of environmental physics. Arnold ed., London, $241 \mathrm{p}$.

Monteith J. L., 1965. Evaporation and environment. In " The state and movement of water in living organisms ". Symp. of the Soc. for Exp. Biol., XIX, 205-234. University Press, Cambridge.

Perrier A., 1967. Approche théorique de la microturbulence et des transferts dans les couverts végétaux en vue de l'analyse de la production végétale. La Météorologie, série 5, 4, 527-550.
Perrier A., 1968. Contribution à l'étude des échanges thermiques en biologie végétale. Rev. gén. thermique, 79-80, 721-740.

Perrier A., 1975. Etude physique de l'évapotranspiration dans les conditions naturelles : II - Expressions et paramètres donnant l'évapotranspiration réelle d'une surface mince. Ann. Agron., 26 (2), 105-123. III - Evapotranspiration réelle et potentielle des couverts végétaux. Ann. Agron., 26 (3), 229-243.

Perrier A., 1976. Etude et essai de modélisation des échanges de masse et d'énergie au niveau des couverts végétaux. Thèse Doct. d'Etat, Univ. Paris VI, 236 p.

Perrier A., Itier B., Bertolini J. M., Blanco de Pablos A., 1975. Mesure automatique du bilan d'énergie d'une culture. Exemples d'application. Ann. Agron., 26 (1), 19-40. 